\title{
Some local forms of known convergences of sequence of real valued functions
}

\author{
Doris Doda ${ }^{\dagger}$ and Agron Tato ${ }^{\ddagger}$ \\ †Wisdom University, Albania \\ ¥Tirana Polytechnic University, Department of Mathematics, \\ Albania \\ †email: dorismuca@live.com
}

\begin{abstract}
Using the notions of local uniform and strong local uniform convergence for the sequence of real valued functions or with value in metric space, the class of locally equally and strong locally equally convergences are studied. We are concern to dependence of type of some convergences from the neighborhood of the limit point. The known locally uniformly convergence is a key of some applications of this idea. We can reformulate one type of Arzelà Theorem and find relations of this convergence with quasi-uniformly by segments of Alexandroff convergence. Beside this type of convergence, we focus to another convergence which is nearer the well known $\alpha$-convergence.
\end{abstract}

\section{Introduction}

There are many authors that try to solve some rebuses of relations of pointwise convergence and other convergences. One of this problems is: what conditions we must be added to point-wise convergence of continuous functions to preserve continuity? This problem is resolve for the first time from Arzelà [1] in 1883. There are more than hundred years that this issue has intrigued many authors. Along the way to finding some convergences in order to relativize recent convergences of Papanastassiou [10] just like Das and Papanastssiou [3], we come up with notions of locally uniformly convergence which is weaker as uniformly equally and extend the most of the 
their properties. The same situation is between the strong uniformly locally equally convergence with $\alpha$-uniformly equally convergence. Using the notion of the exhaustive family given from Gregoriades and Papanastassiou [2] and the notion of $\alpha$-convergence (otherwise continuous convergence or "stetige Konvergenz") has been known by the beginning of the 20th century from work of Stoilov [12] and Arens [6] in 1950s, in the third section, we have present the notion of strong uniformly locally convergence which is stronger then $\alpha$-convergence but weaker then uniform convergence. One special case of strong uniformly locally convergence, denote $\alpha^{*}$-convergence is discussed. By means of uniformly local convergence in real valued functions, in the section fourth, we give any variant of the type of Arzelà Theorem and one equivalent claim in the Alexandroff Theorem. In the fifth section, Das and Papanesstassiu [3] give some very interesting convergences as equally and $\alpha$ equally convergences, follow them we focus in some similar propositions for the uniform locally equally an strong uniformly locally equally sequences.

\section{Notation and terminology}

Let us begin with some comments and notations. With $X$ and $Y$ we mean metric spaces, unless stated otherwise. If it is not mentioned explicitly the symbol $d$ stands for the metric on $X$ and the symbol $p$ for the metric on $Y$.

If $x$ is a member of $X$ and $\delta$ is a positive number, with $S(x, \delta)$ we mean the (open) ball of radius $\delta$, i.e. $S(x, \delta)=\{y \in X / d(x, y)<\delta\}$. Also, if $X$ and $Y$ are metric spaces, as usual, we denote with $C(X, Y)$ the set of all continuous functions from $X$ to $Y$.

We now recall the notion of exhaustiveness [3] which is close to the notion of equi-continuity.

Definition 2.1. Let $(X, d),(Y, p)$ be metric spaces, $x \in X, \mathcal{F}$ be a family of functions from $X$ to $Y$ and $f_{n}: X \rightarrow Y, n \in \mathbb{N}$.

(1) If $\mathcal{F}$ is infinite, we call the family $\mathcal{F}$ exhaustive at $x$ if for each $\varepsilon>0$ there exists $\delta>0$ and $A$ finite subset of $\mathcal{F}$ such that: for each $y \in S(x, \delta)$ and for each $f \in F \backslash A$ we have that $p(f(x), f(y))<\varepsilon$.

(2) In case where $\mathcal{F}$ is finite we define $\mathcal{F}$ to be exhaustive at $x$ if each member of $\mathcal{F}$ is continuous function at $X$.

(3) $\mathcal{F}$ is called exhaustive if $\mathcal{F}$ is exhaustive at every $x \in X$.

(4) The sequence $\left(f_{n}\right)_{n \in \mathbb{N}}$ is called exhaustive at $x$ if for all $\varepsilon>0$ there exist $\delta>0$ and $n_{0} \in \mathbb{N}$ such that for all $y \in S(x, \delta)$ and all $n \geq n_{0}$ we have that $p\left(f_{n}(y), f_{n}(x)\right)<\varepsilon$.

(5) The sequence $\left(f_{n}\right)_{n \in \mathbb{N}}$ is called exhaustive if it is exhaustive at every $x \in X$. 
Notice that in the most interesting cases where $\left(f_{n}\right)_{n \in \mathbb{N}}$ is a sequence of functions for which $f_{n} \neq f_{m}$ for $n \neq m$, then the family $\mathcal{F}=\left\{f_{n} / n \in \mathbb{N}\right\}$ is exhaustive at some $x_{0} \in X$ if and only if the sequence $\left(f_{n}\right)_{n \in \mathbb{N}}$ is exhaustive at $x_{0}$.

Proposition 2.2. [2] Let $(X, d),(Y, p)$ be metric spaces, $x \in X, \mathcal{F}$ a family of functions from $X$ to $Y$ and $f_{n}: X \rightarrow Y, n \in \mathbb{N}$.

(1) $\mathcal{F}$ is equi-continuous at $x$ if and only if $\mathcal{F}$ is exhaustive at $x$ and for each $f \in \mathcal{F}, f$ is continuous at $x$.

(2) The family $\left\{f_{n} / n \in \mathbb{N}\right\}$ is equi-continuous at $x$ if and only if the sequence $\left(f_{n}\right)_{n \in \mathbb{N}}$ is exhaustive at $x$ and each $f_{n}$ is continuous at $x$.

In [2] is given an example that suggests that there exists an exhaustive sequence (similarly family) which contains non continuous functions.

Let we recall the definition of $\alpha$-converges. Let $f, f_{n}, n \in \mathbb{N}$ be functions from $X$ to $Y$. The sequence $\left(f_{n}\right)_{n \in \mathbb{N}}$ is $\alpha$-convergent to $f$ if for each $x \in X$ and for each sequence $\left(x_{n}\right)_{n \in \mathbb{N}}$ of points of $X$ converging to $x$, the sequence $\left(f_{n}\left(x_{n}\right)\right)_{n \in \mathbb{N}}$ converges to $f(x)$.

We shall write $f_{n} \stackrel{\alpha}{\rightarrow} f$ to denote that $\left(f_{n}\right)_{n \in \mathbb{N}}$ is $\alpha$-convergent to $f$. Also we will keep the analogous notation about point-wise and uniform convergence, i.e., we will denote them with $f_{n} \stackrel{p w}{\longrightarrow} f$ and $f_{n} \stackrel{u}{\rightarrow} f$, respectively.

It is obvious that $\alpha$-convergence is stronger than point-wise convergence.

The usual convergences such as point-wise and uniform do not require a topology for the domain space. However a topology is needed for $\alpha$ convergence.

Let $X$ be a non-empty set. By function on $X$, we mean a real valued function on $X$. Let $\Phi$ be an arbitrary class of functions defined on $X$. Then we have the following definitions.

Definition 2.3. [10] A sequence of functions $\left(f_{n}\right)$ in $\Phi$ is said to converge uniformly equally to a function $f$ in $\Phi$ (written as $f_{n} \stackrel{\text { u.e. }}{\longrightarrow} f$ ) if there exists a sequence $\left(\varepsilon_{n}\right)_{n \in \mathbb{N}}$ of positive reals converging to zero and a natural number $n_{0}$ such that the cardinality of the set

$$
\left\{n \in \mathbb{N}:\left|f_{n}(x)-f(x)\right| \geq \varepsilon_{n}\right\}
$$

is at most $n_{0}$, for each $x \in X$.

Definition 2.4. [11] A sequence of functions $\left(f_{n}\right)$ in $\Phi$ is said to converge equally to a function $f$ in $\Phi$ (written as $f_{n} \stackrel{e_{i}}{\rightarrow} f$ ) if there exists a sequence $\left(\varepsilon_{n}\right)_{n \in \mathbb{N}}$ of positive reals converging to zero and a natural number $n(x)$ satisfying $\left|f_{n}(x)-f(x)\right|<\varepsilon_{n}$ for all $n>n(x)$. 
Definition 2.5. Let $(X, d)$ be a metric space and $f, f_{n}: X \rightarrow \mathbb{R}, n \in \mathbb{N}$. Then $\left(f_{n}\right)$ converges $\alpha$-uniformly equally to $f$ (written $f_{n} \stackrel{\alpha-u . e}{\rightarrow} f$ ) if there exists a sequence $\left(\varepsilon_{n}\right)_{n \in \mathbb{N}}$ of positive reals converging to zero and an $n_{0} \in \mathbb{N}$ such that $\left|\left\{n \in \mathbb{N}:\left|f_{n}\left(x_{n}\right)-f(x)\right| \geq \varepsilon_{n}\right\}\right| \leq n_{0}$ for each $x \in X$ and $x_{n} \rightarrow x$.

\section{$3 \delta_{a}, \delta, \alpha^{*}$-convergences}

Definition 3.1. [13] Let $(X, d),(Y, p)$ be metric spaces, $x \in X$ and $f_{n}, f$ : $X \rightarrow Y$. Function $f(x)$ is a locally uniformly limit or $\delta$-limit of the sequence $\left(f_{n}\right)_{n \in \mathbb{N}}$ if for each $\varepsilon>0$, there exist $n_{0}(\varepsilon, x) \in \mathbb{N}$ and $\delta>0$ such that for each $n \geq n_{0}$, and $y \in S(x, \delta)$ we have $p\left(f_{n}(y), f(y)\right)<\varepsilon$.

So we can say that $\left(f_{n}\right)_{n \in \mathbb{N}}$ converges locally uniformly to $f(x)$ or short is $\delta$-convergent to $f(x)$.

This convergence in uniformly on one open neighborhood of a point. So the function $f(x)=x^{n}$ if we see only $[0,1[$ is $\delta$-convergent in this interval.

Proposition 3.2. Let $f_{n}: X \rightarrow Y$ be a sequence that is $\delta$-convergent to a function $f: X \rightarrow Y$ for each $x \in X$. Then the sequence $f_{n}$ converges point-wise to $f$ for each $x \in X$.

Proof. The proof is clear by the Definition (3.1).

The following statements represent some the main feature of local uniform convergence which define its position in respect with point-wise and uniform convergence.

Proposition 3.3. [13] Let $(X, d),(Y, p)$ be metric spaces, $x \in X$ and $f_{n}, f$ : $X \rightarrow Y$.

If the sequence $f_{n}(x)$ is exhaustive and $\delta$-converges to the function $f(x)$. Then the function $f(x)$ is continuous in this point.

If the member of the sequence $f_{n}(x)$ are continuous functions, of course, the limit function is continuous and in the case when the sequence $f_{n}(x)$ is not exhaustive.

Proposition 3.4. Let $(X, d),(Y, p)$ be metric spaces, $x \in X$ and $f_{n}, f: X \rightarrow$ $Y$. The space $X$ is compact and the sequence $f_{n}, \delta$-converges to the function $f$. Then the sequence $f_{n}$ converges uniformly to this function.

Among others, we have also found a definition "of the type $\varepsilon, \delta$ " for $\alpha$ convergence with little restrictions and we name it strong locally uniformly convergent. 
Definition 3.5. Let $(X, d),(Y, p)$ be metric spaces, $x \in X$ and $f_{n}, f: X \rightarrow$ $Y$. We say that sequence $\left(f_{n}(x)\right)_{n \in \mathbb{N}}$ is strong locally uniformly convergent (or short $\delta_{a}$-convergent) to $f(x)$ if for every $\varepsilon>0$ and $x \in X$, there exist $n_{0}(\varepsilon, x) \in \mathbb{N}$ and $\delta>0$ such that for $n \geq n_{0}(\varepsilon, x)$ and $y \in S(x, \delta)$ we have $p\left(f_{n}(y), f(x)\right)<\varepsilon$.

We can profit some properties of $\alpha$-convergence for $\delta_{a}$-convergence with other proofs.

Proposition 3.6. In the conditions of the above preposition the sequence $f_{n}: X \rightarrow Y$ is $\delta_{a}$-convergent to the function $f(x)$. Then

a) Sequence $\left(f_{n}(x)\right)_{n \in \mathbb{N}}$ is exhaustive,

b) $f(x)$ is continuous at $x \in X$.

It looks like the two concepts $\alpha$-convergence and $\delta_{a}$-convergence are equivalent, but it is not. If we fix the metric in $Y$ for the both convergences, then $\delta_{a}$-convergence is stronger that $\alpha$-convergence. In reality, the concept of $\alpha$ convergence used by Kelley ([7] p. 241, M) about nets on topology and two convergence $x_{n} \stackrel{\sigma_{1}}{\rightarrow} x$ and $f_{m}\left(x_{n}\right) \stackrel{\sigma_{2}}{\rightarrow} f(x)$ are considered quite different. If we limit ourselves only to the case when two indexes $n$ and $m$ of the first and the second convergence are comparable on $\mathbb{N}$ then we have a strong $\alpha^{*}$ convergence, that is: we say that the sequence $\left(f_{n}\right)$ is $\alpha^{*}$-convergent on $X$ if for every $\varepsilon>0$ and $x \in X$ there exists a number $n_{1} \in \mathbb{N}$ such that for $n>n_{1}$ the sequence $x_{n} \rightarrow x$ and there exists a number $n_{2} \in \mathbb{N}$ such that for $n>n_{2}$ $f_{n}\left(x_{n}\right) \rightarrow f(x)$.

Proposition 3.7. Let $(X, d),(Y, p)$ be metric spaces, $x \in X$ and $f_{n}, f: X \rightarrow$ $Y$. The following are equivalent:

1. Let be $\varepsilon>0$ and $x \in X$, there exist $n_{0}(\varepsilon, x) \in \mathbb{N}$ and $\delta>0$ such that for $n \geq n_{0}(\varepsilon, x)$ and $y \in S(x, \delta)$ we have $p\left(f_{n}(y), f(x)\right)<\varepsilon$,

2. The sequence $\left(f_{n}(x)\right)_{n \in \mathbb{N}} \alpha^{*}$-converges to $f(x)$.

Proof. $(1) \Rightarrow(2)$. Let us consider the convergence $x_{n} \rightarrow x$, that is, for every $\delta_{1}>0$, there exists $n_{1}\left(\delta_{1}\right) \in \mathbb{N}$ such that for $n>n_{1}(\delta) \Rightarrow d\left(x_{n}, x\right)<\delta_{1}$ or $x_{n} \in S\left(x, \delta_{1}\right)$. It follows that for every $\varepsilon>0$ there exists $\delta<\delta_{1}$ and $n_{2} \geq \max \left(n_{0}, n_{1}\right)$ such that when $x_{n} \in S(x, \delta) \Rightarrow d\left(f_{n}\left(x_{n}\right), f(x)\right)<\varepsilon$. It is proved that $f_{n}$ is $\alpha$-convergent to $f(x)$.

$(2) \Rightarrow(1)$. Let us assume that $f_{n}(x)$ is not convergent according first statement. Hence there exists one $\varepsilon_{0}>0$ such that for $x \in X$ and $\bar{n}\left(\varepsilon_{0}, x\right) \in$ $\mathbb{N}, \delta>0$ such that for $n \geq \bar{n}\left(\varepsilon_{0}, x\right)$ and $y \in S(x, \delta) \Rightarrow p\left(f_{n}(y), f(x)\right) \geq \varepsilon_{0}$. By the second statement when $x_{n} \rightarrow x$ there exists one $\delta>0$ such that $n>n_{1}(\delta) \Rightarrow d\left(x_{n}, x\right)<\delta$ but $f_{n}(x)$ don't converges to $f(x)$ according Definition 3.1. That contradicts (2). 


\section{On Arzelà and Alexandroff Theorems}

The proof of first theorem is based by Arzelà on the concept of quasi-uniform convergence to a compact interval of $\mathbb{R}$. Let we compare the locally uniformly convergence with quasi-uniform convergence.

Definition 4.1. [1] Let $\left(f_{n}\right)_{n \in \omega}$ be a sequence of real-valued continuous function defined on a compact interval $[a, b]$ and $f$ be a real-valued function defined on $[a, b]$. The sequence $\left(f_{n}\right)_{n \in \omega}$ is said to converge to $f$ quasi-uniformly by segments(a tratti) on $[a, b]$ if

(i) $\left(f_{n}\right)_{n \in \omega}$ converges point-wise to $f$,

(ii) for every $\varepsilon>0$ and every $n_{0}$ there exist a finite number of naturals $n_{1}, \ldots, n_{t} \geq n_{0}$ and closed intervals $S_{n_{1}}, \ldots, S_{n_{t}}$ such that the union of their interiors cover $[a, b]$, and

$$
\forall x \in S_{n_{i}},\left|f_{n_{i}}(x)-f(x)\right|<\varepsilon, \quad i=1, \ldots, t .
$$

Theorem 4.2. [1] (Arzelà Theorem) Let $\left(f_{n}\right)_{n \in \omega}$ be a sequence of real-valued continuous function defined on a compact interval $[a, b]$ and $f$ be a real-valued function defined on $[a, b]$. Let $\left(f_{n}\right)_{n \in \omega}$ be point-wise convergent to $f$. Then $f$ is continuous if and only if $\left(f_{n}\right)_{n \in \omega}$ is said to converge to $f$ quasi-uniformly by segments.

Proposition 4.3. Definitions 3.1 and 4.1 in the case of the continuous and real-valued functions are equivalent.

Proof. (3.1) $\Rightarrow$ (4.1) By the Proposition 3.2 we proved the condition (1) of (4.1). Now, since interval $[a, b]$ is the compact set there exists the cover $\left\{S_{m}^{0}\right\}$ of it and from this a finite subcover $S_{n_{1}}^{0}, \ldots, S_{n_{k}}^{0}$ such that $[a, b] \subset \bigcup_{i=1}^{k} S_{n_{i}}^{0}$ and $S_{n_{i}}=S_{n_{i}}^{0} \cap[a, b]$. By the Definition 2.1, for the every $\varepsilon>0$ and $x \in S_{n_{i}}$ there exist $\delta>0, n_{i}\left(\varepsilon, \delta_{i}\right) \in \mathbb{N}$ and $n>n_{i}\left(\varepsilon, \delta_{i}\right)$ such that for every $y \in S\left(x, \delta_{i}\right)$ we have $\left|f_{n_{i}}(y)-f(y)\right|<\varepsilon$. In the case of ball $S_{n_{i}}$ we are in condition of the interval $[a, b]$, so if $\left\{S\left(x, \delta_{x}\right)\right\}$ is a cover of $S_{n_{i}}$ for every $x \in S_{n_{i}}$, we can find a finite subcover $S_{n_{i}} \subset \bigcup_{j=1}^{m} S\left(x_{i}, \delta_{x_{i}}\right)$. For every $x_{i}$ we find a number $n_{i}\left(\varepsilon, \delta_{x_{i}}\right)$. Let $n_{0 i}$ be $\max \left\{n_{i}\left(\varepsilon, \delta_{x_{i}}\right)\right\}$. If we take $i=1, \ldots, k$ than there exist the sequence $n_{01}, \ldots, n_{0 k}>n_{0}$ such that for every $y \in S_{n_{i}}$ we have $\left|f_{n_{i}}(y)-f(y)\right|<\varepsilon$. This prove the condition (4.1).

$(4.1) \Rightarrow(3.1)$ By the Definition 4.1, there exist a finite number of naturals $n_{1}, \ldots, n t \geq n_{0}$ and sequence of closed sets $S_{n_{1}}, \ldots, S_{n_{t}}$ such that $[a, b] \subset$ $\bigcup_{i=1}^{k} S_{n_{i}}^{0}$ and for every $y \in S_{n_{i}}$ we have $\left|f_{n_{i}}(y)-f(y)\right|<\varepsilon$. Let $\varepsilon$ be a positive number and every $x$ such that $x \in[a, b]$ than there exists $S_{n_{j}}^{0}$ such that $x \in S_{n_{j}}^{0}$ and $\left|f_{n_{i}}(x)-f(x)\right|<\varepsilon$, for every $x \in S_{n_{j}}^{0}$ and $n_{j}>n_{0}$. If we take a 
$\delta>0$ such that $x \in S(x, \delta) \in S_{n_{j}}^{0}$ for every $y \in S(x, \delta)$ there exists $n_{j}>n_{0}$ such that $\left|f_{n}(y)-f(y)\right|<\varepsilon$.

We can give another form of Arzelà Theorem.

Theorem 4.4. Let $\left(f_{n}\right)_{n \in \omega}$ be a sequence of real-valued continuous functions defined on a compact interval $[a, b]$ and $f$ be a real-valued function defined on $[a, b]$. Then $f$ is continuous if and only if $\left(f_{n}\right)_{n \in \omega}$ is locally uniformly convergent (short $\delta$-convergent) to $f$.

In 1948 Alexandroff [4] made the following definition:

Definition 4.5. Let $\left(f_{n}\right)_{n \in \omega}$ be a sequence of functions from a topology space $X$ to the metric space $(Y, \rho)$ and let $f: X \rightarrow Y$. Then $\left(f_{n}\right)_{n \in \omega}$ is called Alexandroff convergent to $f$ on $X$, provided it point-wise convergent to $f$, and for every $\varepsilon>0$ and integer $n_{0}$ there exists a countable open covering $\left\{\Gamma_{1}, \Gamma_{2}, \ldots\right\}$ of $X$ and sequence $\left\{n_{k}\right\}$ of positive integers greater than $n_{0}$ such that for each $x_{k}$ we have $\rho\left(f_{n_{k}}, f\right)<\varepsilon$.

We can added the famous Alexandroff Theorem another third equivalent claim and take the proposition.

Definition 4.6. Let $X$ be a topological space and $(Y, \rho)$ metric space. Let $\left\{f_{n}\right\}_{n \in \omega}$ be sequence of continuous functions from $X$ to $Y$ point-wise convergent to a function $f$ from $X$ to $Y$. The following conditions are equivalent:

(i) $f$ is continuous;

(ii) $\left(f_{n}\right)_{n \in \omega}$ Alexandroff convergent to $f$;

(iii) $\left(f_{n}\right)_{n \in \omega}$ is locally uniformly convergent to $f$.

Proof. For the implications (i) $\Rightarrow$ (ii) and (ii) $\Rightarrow$ (i) one can see [4].

(ii) $\Rightarrow$ (iii). Let $\varepsilon>0$ and $x \in X$. From (ii) for this $\varepsilon>0$ and integer $n_{0}$, there exists a countable open covering $\left\{\Gamma_{1}, \Gamma_{2}, \ldots\right\}$ of $X$, that is $X \subseteq \bigcup_{k=1}^{\infty} \Gamma_{k}$ and sequence $\left\{n_{k}\right\}$ of positive integers greater than $n_{0}$ such that for each $n_{k} \geq n_{0}$ we have $\rho\left(f_{n_{k}}, f\right)<\varepsilon$. From the fact that $x \in X$ follows that there exist a $\delta>0$ such that $S(x, \delta) \subset \Gamma_{k_{j}}$. On the other side, for every $y \in S(x, \delta)$ and $n_{k_{j}} \geq n_{0} \Rightarrow \rho\left(f_{n_{k_{j}}}(y), f(y)\right)<\varepsilon$. This prove (iii).

(iii) $\Rightarrow$ (i). Assume that $\left(f_{n}\right)_{n \in \omega}$ is locally uniformly convergent to $f$. Then for every $\varepsilon>0$ and $x \in X$ there exist a $n(\varepsilon) \in \mathbb{N}$ and neighborhood $V_{x}$ such that for every $n>n(\varepsilon)$ and $y \in V_{x}$ we have that $\rho\left(f_{n}(y), f(y)\right)<\frac{\varepsilon}{3}$ and also $\rho\left(f_{n}(x), f(x)\right)<\frac{\varepsilon}{3}$. Since the functions $\left(f_{n}\right)_{n \in \omega}$ are continuous for this $\varepsilon$ there exists a neighborhood $U_{x}$ of the point $x$ such that for every $y \in U_{x}$ and every $n \in \mathbb{N}$ we obtain $\rho\left(f_{n}(y), f_{n}(y)\right)<\frac{\varepsilon}{3}$. It is easy to see that for every $y \in V_{x} \cap U_{x}$ we have

$$
\rho(f(y), f(x)) \leq \rho\left(f(y), f_{n}(y)\right)+\rho\left(f_{n}(y), f_{n}(x)\right)+\rho\left(f_{n}(x), f(x)\right)<\varepsilon
$$


Bartle [5] in 1955 investigate the continuity of limits of nets of real-valued continuous functions and reformulated the above definitions of Arzelà and Alexandroff.

We can extended these definition in case of net of real-valued function on one set $X$.

Definition 4.7. (i) Let $\left(f_{\alpha}\right)_{\alpha \in \Lambda}$ be a net of real-valued functions on an arbitrary set $X$ and $f: X \rightarrow Y$. Then $\left(f_{\alpha}\right)_{\alpha \in \Lambda}$ is said to converge to $f$ quasi-uniformly on $X$, provided it point-wise converges to $f$, and for every $\varepsilon>0$ and $\alpha_{0}$ there exists a finite number of indices $\alpha_{1}, \ldots, \alpha_{n} \geq \alpha_{0}$ such that for each $x \in X$ at least one of the following inequalities holds:

$$
\left|f_{\alpha_{i}}(x)-f(x)\right|<\varepsilon, \quad i=1, \ldots, n
$$

(ii) in these condition $\left(f_{\alpha}\right)_{\alpha \in \Lambda}$ is said to converge to $f$ locally uniformly to $x \in X$ if for every $\varepsilon>0$ there exist a $\alpha_{0} \in \Lambda$ and a neighborhood $V_{x}$ such that for $\alpha_{i} \geq \alpha_{0}$ and $y \in V_{x}$ we have $\rho\left(f_{\alpha_{i}}(y), f(y)\right)<\varepsilon$

Following [9], [8], in which paper of them, they extended the Arzelà Theorem for nets of functions with domain is $k$-space and with values into a metric space. We can reformulate the proposition:

Theorem 4.8. Let $X$ be a k-space and $(Y, \rho)$ be a metric space. Let $\left(f_{\alpha}\right)_{\alpha \in \Lambda}$ be a net of continuous functions from $X$ to $Y$ which point-wise converges to a function $f$ from $X$ to $Y$. The following condition are equivalent:

(i) $f$ is continuous;

(ii) $\left(f_{\alpha}\right)_{\alpha \in \Lambda}$ converges to $f$ quasy-uniformly on compacta (i.e. in on compact sets)

(iii) $\left(f_{\alpha}\right)_{\alpha \in \Lambda}$ converges to $f$ locally uniformly on locally compact space.

Proof. (i) $\Rightarrow$ (ii). See Theorem 3.4. in [5] and (ii) $\Rightarrow$ (i) see [9].

(i) $\Rightarrow$ (iii). Let $f$ be a continuous limit of point-wise convergence of $\left(f_{\alpha}\right)_{\alpha \in \Lambda}$. That is for every $\varepsilon>0$ there exists the $\alpha_{0}$ such that for every $\alpha(x) \geq \alpha_{0}$ we have $\rho\left(f_{\alpha(x)}(x), f(x)\right)<\frac{\varepsilon}{3}$. Since the function $f$ and $f_{\alpha}(y)$ are continuous for every $\varepsilon>0$ there exists a neighborhood $S\left(x, \delta_{1}\right)$ of the point $x$ such that for $y \in S\left(x, \delta_{1}\right)$ follows that $\rho(f(x), f(y))<\frac{\varepsilon}{3}$ and $\rho\left(f_{\alpha}(y), f_{\beta}(y)\right)<$ $\frac{\varepsilon}{3}$ for every $\alpha$ and $\beta$.

Let $N_{x}=\left\{y: \rho\left(f_{\alpha(x)}(y), f(y)\right)<\frac{\varepsilon}{3}\right\}$. From the continuity of $f$ and $f_{\alpha}$ comes that $N_{x}$ is an open set which contains $x$. Let $S\left(x, \delta_{2}\right)$ be a ball that $S\left(x, \delta_{2}\right) \subset N_{x}$. As the space $X$ is locally compact then point-wise convergence of $\left(f_{\alpha}\right)_{\alpha \in \Lambda}$ is uniform and we can choose an $\alpha^{*}$ such that for $\alpha, \beta \geq \alpha^{*}$ and $y \in S(x, \delta)$, where $\delta=\min \left(\delta_{1}, \delta_{2}\right)$ it holds

$$
\rho\left(f_{\alpha}(y), f(y)\right) \leq \rho\left(f_{\alpha}(y), f_{\beta}(x)\right)+\rho\left(f_{\beta}(x), f(x)\right)+\rho(f(x), f(y))<\varepsilon .
$$

The implication (iii) $\Rightarrow$ (i) repeated the Theorem 4.4. 


\section{Some new forms of the equally convergences}

We can continue the work [3] of the authors R. Das, N. Papanastassiou. They develop the concept of uniformly equally convergence as well as discrete convergence $\alpha$-uniformly equally convergence, in order to profit same important characterization of compactness in metric space. In the following we can do the same think with other tools.

Definition 5.1. A sequence of functions $\left(f_{n}\right)$ in $\Phi$ is said to converge uniformly locally equally to a function $f$ in $\Phi$ (written as $f_{n} \stackrel{\text { u.l.e. }}{\longrightarrow} f$ ) for every $x \in X$ if there exists a sequence $\left(\varepsilon_{n}\right)_{n \in \mathbb{N}}$ of positive real numbers converging to zero, a neighborhood $V_{x}$ and a natural number $n_{0}(x, V)$ such that the cardinality of the set $\left\{n \in \mathbb{N}:\left|f_{n}(y)-f(y)\right| \geq \varepsilon_{n}\right\}$ is at most $n_{0}(x, V)$, for each $y \in V_{x}$.

It is clear that if the sequence is uniformly equally then it is uniformly locally equally. The following example show that conversely there don't holds.

Example 5.2. Let $f_{n}=x^{n}$ be a sequence of functions determine on the interval $[0,1$ and to testify that from the uniformly locally equally convergence don't derive the uniformly equally convergence. Let $\left(\frac{1}{n}\right)$ be a sequence converging to zero and $0 \leq x<1$. Put $\delta=\min \{|x|,|1-x|\}$ and $y \in] x-\delta, x+\delta[$. Let we prove that the cardinal of the set $\left\{n \in \mathbb{N}: y^{n} \geq \frac{1}{n}\right\}$ is finite number. We know that $n y^{n} \rightarrow 0$ if $|y|<1$ and $n \rightarrow \infty$. Then for every $\varepsilon>0$ and for $\varepsilon=1$, there exists a natural number $n_{0}$ such that for $n>n_{0}$ we have $n y^{n} \leq 1$. Since $y^{n} \geq \frac{1}{n}$ holds for at most $n \leq n_{0}$. In paper [3] (Example 4.9.) is proved that this sequence is not uniformly equally to this interval.

Proposition 5.3. On the compact metric space the convergences of uniformly equally and uniformly locally equally coincide.

Proof. $(2.3) \Rightarrow(5.1)$ By the definitions.

$(5.1) \Rightarrow(2.3)$. By the Definition 2.3, there exists the sequence of positive real numbers converging to zero and $f_{n} \stackrel{u . l}{\longrightarrow} f$, that is, for every $x \in X$ there exist a $\delta_{x}>0$ and $n\left(x, \delta_{x}\right) \in \mathbb{N}$ such that for every $y \in B\left(x, \delta_{x}\right)$ we have

$$
\left|\left\{n \in \mathbb{N}:\left|f_{n}(y)-f(y)\right| \geq \varepsilon_{n}\right\}\right|<n\left(x, \delta_{x}\right) .
$$

Let the set of $\left\{B\left(x, \delta_{x}\right)\right\}$ an open cover of $X$ and there exists a subcover of it such that $X \subset \bigcup_{k=1}^{m} B\left(x_{k}, \delta_{x_{k}}\right)$. Now, if $x \in X$, there exists a ball $B(x, \delta)$ such that $x \in B(x, \delta) \subset B\left(x_{k}, \delta_{x_{k}}\right)$. By the inequality (5.1) we obtain

$$
\left|\left\{n \in \mathbb{N}:\left|f_{n}(y)-f(y)\right| \geq \varepsilon_{n}\right\}\right|<n\left(x_{k}, \delta_{x_{k}}\right) .
$$


We put $n_{0}=\max \left\{n\left(x_{1}, \delta_{x_{1}}\right), \ldots, n\left(x_{m}, \delta_{x_{m}}\right)\right.$ and for every $y \in X$ the cardinal of the set $\left|\left\{n \in \mathbb{N}:\left|f_{n}(y)-f(y)\right| \geq \varepsilon_{n}\right\}\right|$ is at most $n_{0}$.

We can modify the Theorem 4.12 in [3] and profit another important characterization of compact space through convergent sequences. First, we give the definition of the lattice. Let we recall that the function class $\Phi$ on $X$ is called lattice if $\Phi$ contains all constants and $f, g \in \Phi$ implies $\max (f, g) \in \Phi$ and $\min (f, g) \in \Phi$.

Theorem 5.4. A metric space $(X, d)$ is compact if and only if the uniformly locally equally convergence of the sequence $\left(f_{n}\right)$ of real functions from a lattice $\Phi$ on $X$ convergent to zero implies that uniformly equally convergence of the sequence $\left(f_{n}\right)$ to zero function.

Proof. The case when $X$ is compact space it is seen in the above preposition. For the prove of inverse, suppose that $(X, d)$ is not a compact metric space. Being that $X$ is not compact, there exists a sequence $\left\{x_{k}\right\}$ of distinct points of $x$ such that, there exists one no convergent subsequence of $\left\{x_{k}\right\}$. Since every point of the set $\left\{x_{1}, \ldots, x_{k}, \ldots\right\}$ in an isolated point of this set, there exists $\delta_{k}>0$ and $\delta_{k} \rightarrow 0, k=1,2, \ldots$ such that constructed open balls

$$
B\left(x_{k}, \delta_{k}\right)=\left\{x \in X: d\left(x, x_{k}\right) \leq \delta_{k}\right\} \quad k=1,2, \ldots
$$

are pair-wise disjoint. Then the set $H=\bigcup_{k=1}^{\infty} B\left(x_{k}, \delta_{k}\right)$ is a open. Define a sequence $\left(f_{p}\right)_{p \in \mathbb{N}}$ of real valued functions on $X$.

$$
f_{p}(x)= \begin{cases}0, & \text { if } x \in X \backslash H \\ \frac{n^{p}}{p !} & \text { if } x \in H\end{cases}
$$

If $x \in H$ then there exists $B\left(x_{k}, \delta_{k}\right)$ such $x \in B(x, \delta) \subset B\left(x_{k}, \delta_{k}\right)$ and for every $y \in B(x, \delta)$ there exists a sequence of real positive numbers $\varepsilon_{p}=\frac{1}{p} \rightarrow 0$ as $p \rightarrow \infty$ such that $\left|\left\{n \in \mathbb{N}:\left|f_{p}(x)\right| \geq \varepsilon_{n}\right\}\right| \leq p$ for every $p$ and $x \in X$. The sequence $\left(f_{p}\right)_{p \in \mathbb{N}}$ converges uniformly locally equally but not uniformly equally because $p$ is not bounded.

It is evident that point-wise convergence is equally convergence. Let we relativize this definition to profit the definition of locally equally.

Definition 5.5. A sequence of functions $\left(f_{n}\right)$ in $\Phi^{\prime}$ is said to converge locally equally to a function in $\Phi^{\prime}$ (written as $f_{n} \stackrel{\text { l.e. }}{\rightarrow} f$ ) for every $x \in X$ if there exists a sequence $\left(\varepsilon_{n}\right)_{n \in \mathbb{N}}$ of positive reals converging to zero, a neighborhood $V_{x}$ and a natural number $n\left(x, V_{x}\right)$ such that for every $y \in V_{x}$ we have $\left|f_{n}(x)-f(x)\right|<$ $\varepsilon_{n}$ for all $n>n\left(x, V_{x}\right)$. 
Note. By the definitions we see that equally convergence is locally equally convergence.

Example 5.6. In the Example 4.4. of the reference [3] is given the idea to prove that. There is a sequence $\left(f_{n}\right)$ equally convergent but no locally equally convergent. Define as the characteristic function of the intervals $\left[n, n+\frac{1}{n}\right], n \in \mathbb{N}$ and zero at all other points of set $[1, \infty[$. It is easy to see that if $\left(\varepsilon_{n}\right)_{n \in \mathbb{N}}$ is a sequence of positive reals converging to zero then $\left|\left\{n \in \mathbb{N}:\left|f_{n}(x)\right| \geq \varepsilon_{n}\right\}\right| \leq 1$. It turns out that for $n>2$ comes that $\left|f_{n}(x)\right|<\varepsilon_{n}$ for every $x \in\left[1, \infty\left[\right.\right.$. The sequence $\left(f_{n}\right)$ is equally convergent. On the other side, for every $x \in\left[n, n+\frac{1}{n}\right]$, there exists one $n_{0}$ that for $n>n_{0}$ $f_{n}(x)=1>\varepsilon_{n}$. The sequence $\left(f_{n}\right)$ is not locally equally convergent.

It easy to prove the following proposition.

Proposition 5.7. The equally convergence coincide with the uniform convergence in a case when the space $X$ is a compact.

Proof. It is evident the fact that uniform convergence implies equally convergence. For the inverse direction, it is enough to prove that from equally convergence derives the point-wise convergence on $X$. By the Definition 2.4 there exists a sequence $\left(\varepsilon_{n}\right)_{n \in \mathbb{N}}$ of positive reals converging to zero and a natural number $n(x)$ satisfying $\left|f_{n}(x)-f(x)\right|<\varepsilon_{n}$ for all $n>n(x)$. Let $\varepsilon$ be a whatever positive number. There exists a natural number $n_{1}$ such that for every $n \in \mathbb{N}$ and $n>n_{1}$ we have that $\varepsilon_{n}<\varepsilon$. If we put $n_{2}(x)=\max \left\{n(x), n_{1}\right\}$ then for $n>n_{2}(x)$ we obtain $\left|f_{n}(x)-f(x)\right|<\varepsilon_{n}$ for every $\varepsilon>0$ and $x \in X$. This prove the proposition.

Proposition 5.8. Let $X$ be a locally compact space and the sequence $\left(f_{n}\right)$ is equally convergent. Then the sequence $\left(f_{n}\right)$ is also locally equally convergent.

Proof. By the Definition 5.5, for every $x \in X$, there exists a sequence $\left(\varepsilon_{n}\right)_{n \in \mathbb{N}}$ of positive reals converging to zero and a natural number $n(x)$ satisfying $\left|f_{n}(x)-f(x)\right|<\varepsilon_{n}$ for all $n>n(x)$. Let $S(x, \delta)$ be a neighborhood of point $x$ on $X$. Since $X$ is locally compact, the set $\overline{S(x, \delta)}$ is a compact neighborhood of the point $x$. By the above Proposition (5.7), equally convergence on the set $X \cap \overline{S(x, \delta)}$ implies the uniform convergence in this set. Therefore, for every $\varepsilon>0$ and for every $\left(\varepsilon_{n}\right)_{n \in \mathbb{N}}$ of positive reals converging to zero there exists $n_{0}(\delta)$ such that for every $n \in \mathbb{N}$ and $n>n_{0}(\delta)$ we have $\left|f_{n}(y)-f(y)\right|<\varepsilon_{n}$ for every $y \in S(x, \delta)$. By the fact that $x$ and $\delta$ are arbitrary the sequence converges locally equally.

By following definition we can prove almost all properties and result of $\alpha$-uniformly equally convergence. If we take in account the that all of this 
has the same idea with above propositions then we will be limited only with two of them.

Definition 5.9. Let $(X, d)$ be a metric space and $f, f_{n}: X \rightarrow \mathbb{R}, n \in \mathbb{N}$. Then $\left(f_{n}\right)$ converges $\alpha^{*}$-uniformly locally equally to $f$ (written $f_{n} \stackrel{\alpha^{*}-\text { ul.e }}{\longrightarrow}$ $f)$ or strong uniformly locally equally if there exists a sequence $\left(\varepsilon_{n}\right)_{n \in \mathbb{N}}$ of positive reals converging to zero and $\delta>0$ as well as an $n_{0}(x, \delta) \in \mathbb{N}$ such that $\left|\left\{n \in \mathbb{N}:\left|f_{n}(y)-f(x)\right| \geq \varepsilon_{n}\right\}\right| \leq n_{0}(x, \delta)$ for each $y \in S(x, \delta)$.

It is easy to see that from definition of $\alpha^{*}$-u.l.e. convergence implies both $\alpha^{*}$-convergence and l.e. convergence.

Theorem 5.10. Let $(X, d)$ be a metric space and $f, f_{n}: X \rightarrow \mathbb{R}, n \in \mathbb{N}$. If the sequence $\left(f_{n}\right)$ converges uniformly to zero function, then the sequence $\left(f_{n}\right)$ converges $\alpha^{*}$-u.l.e. to the zero function.

Proof. Since $f_{n} \stackrel{u}{\rightarrow} f(x)=0$, there exists a sequence $\left(\varepsilon_{n}\right)_{n \in \mathbb{N}}$ of positive reals converging to zero and $n_{0} \in \mathbb{N}$ such that for all $n \geq n_{0}$ and for $y \in X$. From the fact that the sequence $\alpha^{*}$-u.l.e. converges if and only if that for every $y \in S(x, \delta)$ and $n_{0} \in \mathbb{N}$, we have

$$
\left|\left\{n \in \mathbb{N}:\left|f_{n}(y)-(f(x)=0)\right| \geq \varepsilon_{n}\right\}\right| \leq n_{0} .
$$

Hence $f_{n} \stackrel{\alpha^{*}-\text { u.l.e }}{\longrightarrow} 0$.

Theorem 5.11. Let $(X, d)$ be a compact space and $f, f_{n}: X \rightarrow \mathbb{R}, n \in \mathbb{N}$. Then $f_{n} \stackrel{\alpha^{*}-\text { u.l.e }}{\longrightarrow} f \Rightarrow f_{n} \stackrel{u}{\rightarrow} f$.

Proof. It follows from the fact that $f_{n} \stackrel{\alpha^{*}-\text { u.l.e }}{\longrightarrow} f \Rightarrow f_{n} \stackrel{\text { u.l.e. }}{\longrightarrow} f$ but in this compact space $f_{n} \stackrel{\text { u.l.e. }}{\longrightarrow} f \Rightarrow f_{n} \stackrel{u}{\rightarrow} f$.

\section{References}

[1] C. Arzelà, Intorno alla continuitá della somma di infinitá di funzioni continue, Rend. R. Accad. Sci. Istit. Bologna (1883/1884), 79-84.

[2] V. Gregoriades, N. Papanastassiou, "The notion of exhaustiveness and Ascoli-type theorems", Topology and its Applications 155 (2008), 11111128.

[3] R. Das, N. Papanastassiou, Some types of convergence of sequences of real valued functions, Real Anal. Exchange 28 (2) (2002/2003) 1-16. 
[4] P.S. Alexandroff, Einführung in die Mengenlehre und die Theorie der reellen Funktionem, Deutsch Verlag Wissenschaft, 1956, translated from the 1948, Russian edition.

[5] R.G. Bartle, On compactness in functional analysis, Trans. Amer. Math. Soc. 79 (1955), 35-57.

[6] R.F. Arens, A topology for spaces of transformations, Ann. of Math. (2) 47 (3) (1946), 480-495.

[7] Kelley, J., General topology, Springer-Verlag, 1975.

[8] L. Holà, T. Šalát, Graph convergence, uniform, quasi-uniform and continuous convergence and some characterizarions of compactness, Acta Math. Unic. Comenian, 54-55 (1998), 121-132.

[9] Agata Caserta, Giuseppe Di Maio , Lubica Holá, Arzelà's Theorem and strong uniform convergence on bornologies, J. Math. Anal. Appl. 371 (2010), 384-392.

[10] N. Papanastassiou, On a new type of convergence of sequences of functions, submitted.

[11] A. Császár and M. Laczkovich, Discrete and equal convergence, Studia Sci. Math. Hungar., 10 (1975), 463-472.

[12] S. Stoilov, Continuous convergence, Rev. Math. Pures Appl. 4 (1959), 341-344.

[13] D. Doda, A. Tato, Some local uniform convergences and its applications on Integral theory, 2018. (submitted) 